\title{
Another view on the prediction of outcomes in patients with community-acquired pneumonia
}

\section{To the Editors:}

At least two clinical rules for predicting short- and long-term mortality in patients with community-acquired pneumonia (CAP) have been successfully validated: the Pneumonia Severity Index (PSI), and the CURB-65 score (confusion, urea $>7 \mathrm{mmol} \cdot \mathrm{L}^{-1}$, respiratory frequency $\geqslant 30$ breaths $\cdot \mathrm{min}^{-1}$, systolic blood pressure $<90 \mathrm{mmHg}$ or diastolic blood pressure $\leqslant 60 \mathrm{mmHg}$ and age $\geqslant 65 \mathrm{yrs}$ ) and its modifications. The biomarker procalcitonin (PCT) has received much attention as another tool to predict outcomes and, possibly, stratify patients according to treatment settings. Recently, we found that PCT might carry an additional predictive potential for mortality across the clinical prediction score CRB-65 (confusion, respiratory frequency $\geqslant 30$ breaths $\cdot \mathrm{min}^{-1}$, systolic blood pressure $<90 \mathrm{mmHg}$ or diastolic blood pressure $\leqslant 60 \mathrm{mmHg}$, and age $\geqslant 65$ yrs) [1]. SCHUETz et al. [2] recently reported that PCT performs poorly as a predictor of mortality and does not increase mortality prediction levels of clinical scores.

There may be several reasons for these conflicting results. One of these reasons may be differences in the populations studied, particularly in the number and type of comorbidities. Another potential bias is the study design, since the study by SHUETZ et al. [2] was primarily designed to guide treatment decisions and not to evaluate outcomes. Finally, the number of patients who received antimicrobial pre-treatment may affect the prognostic predictions. We previously showed that predictive values of PCT are considerably negatively affected by antimicrobial pretreatment [3]. Unfortunately, ScHuETz et al. [2] did not report the number of patients who received such pre-treatment.

In addition, we are tempted to ask whether a different analysis would not lead to divergent conclusions.

First, the authors decided to test predictions based on PCT levels using a four-level tool in order to make decisions on antimicrobial treatment. The use of only one threshold would have been statistically adequate, since deaths were very rare in the low-risk groups (four deaths in the group with $<0.1 \mu \mathrm{g} \cdot \mathrm{L}^{-1}$ PCT and seven deaths in the group with $\left.0.1-0.25 \mu \mathrm{g} \cdot \mathrm{L}^{-1} \mathrm{PCT}\right)$. In fact, there was no linear trend across the four levels; however, figure 1 in that article demonstrates that mortality was twice as high in those with PCT levels $>0.25 \mu \mathrm{g} \cdot \mathrm{L}^{-1}$, which is very close to our findings suggesting that a cut-off of $0.228 \mu \mathrm{g} \cdot \mathrm{L}^{-1}$ separates survivors from nonsurvivors quite confidently.

Secondly, from previous data, it appears that PCT level, alone or in conjunction with clinical scores, predicts mortality best in higher risk classes. This may also be a consequence of very low mortality in low-risk classes, leading to a high probability of missing a small, but possibly significant, difference.
Thirdly, mortality increasingly appears to be a problematic end-point of risk assessment. The authors found that the rate of complications and intensive care unit (ICU) admission, but not mortality, closely followed the four-level risk model of PCT. This may hint at the problem of treatment restrictions in elderly and/or severely disabled patients, also favouring death in lower and intermediate risk classes. Unfortunately, SCHUETZ et al. [2] did not report the number or mortality of patients at highest risk, i.e. those residing in nursing homes or being bedridden. In any case, complications and ICU admission appear to be the more robust end-points for the evaluation of predictive tools of outcome in patients with CAP.

Overall, receiver operating characteristics differed significantly from our findings, which showed PCT as a good predictor of mortality and CRB-65 plus PCT as the best. In the study by SCHUETZ et al. [2], the area under the curve (AUC) of initial PCT was very low (AUC 0.6, 95\% CI 0.52-0.67). Potential reasons for this large difference are numerous, and include characteristics of the population studied, different study design and the number of patients with antimicrobial pretreatment. Such variations challenge the general use of PCT as a predictive tool.

However, different conclusions may be drawn from the study by SCHUETz et al. [2]. A PCT value of $\sim 0.25 \mu \mathrm{g} \cdot \mathrm{L}^{-1}$ identifies patients at increased risk of death. When PCT is used with clinical scores, it might give additional predictive value; in fact, whereas the predictive potential in low risk classes is currently unknown, it seems to be considerable in moderate- and highrisk classes [4]. Having said this, it appears from other studies that pro-adrenomedullin is superior in terms of prediction of prognosis [5-7].

In any case, mortality as end-point should be regarded with caution because of variations in populations studied, comorbidities and possible effects of treatment restrictions, and at least always be interpreted in the light of complication and ICU admission rates. In our view, this seems to be an important message of the study by SCHUETz et al. [2].

\section{S. Krüger*, T. Welte ${ }^{\#}$ and S. Ewig}

*Medical Clinic I, University Clinic RWTH Aachen, Aachen, "Dept of Pneumology, Hanover Medical School, University Clinic Hanover, Hanover, and Thoraxzentrum Ruhrgebiet, Kliniken für Pneumologie und Infektiologie, Ev. Krankenhaus Herne and Augusta Kranken-Anstalt Bochum, Bochum, Germany.

Correspondence: S. Ewig, Thoraxzentrum Ruhrgebiet, Kliniken für Pneumologie und Infektiologie, EVK Herne and 
Augusta-Kranken-Anstalt Bochum, Bergstrasse 26, 44791 Bochum, Germany. E-mail: ewig@augusta-bochum.de

Statement of Interest: Statements of interest for T. Welte and S. Ewig can be found at www.erj.ersjournals.com/site/misc/ statements.xhtml

\section{REFERENCES}

1 Krüger S, Ewig S, Marre R, et al. Procalcitonin predicts patients at low risk of death from community-acquired pneumonia across all CRB-65 classes. Eur Respir J 2008; 31: 349-355.

2 Schuetz P, Suter-Widmer I, Chaudri A, et al. Prognostic value of procalcitonin in community-acquired pneumonia. Eur Respir J 2011; 37: 384-392.

3 Krüger S, Ewig S, Kunde J, et al. Assessment of inflammatory markers in patients with community-acquired pneumonia: influence of antimicrobial pre-treatment: results from the German competence network CAPNETZ. Clin Chim Acta 2010; 411: 1929-1934.

4 Huang DT, Weissfeld LA, Kellum JA, et al. Risk prediction with procalcitonin and clinical rules in community-acquired pneumonia. Ann Emerg Med 2008; 52: 48-58.

5 Christ-Crain M, Morgenthaler NG, Stolz D, et al. Pro-adrenomedullin to predict severity and outcome in community-acquired pneumonia. Crit Care 2006; 10: R96.

6 Huang DT, Angus DC, Kellum JA, et al. Midregional proadrenomedullin as a prognostic tool in community-acquired pneumonia. Chest 2009; 136: 823-831.

7 Krüger S, Ewig S, Giersdorf S, et al. Cardiovascular and inflammatory biomarkers to predict short- and long-term survival in communityacquired pneumonia: results from the German Competence Network, CAPNETZ. Am J Respir Crit Care Med 2010; 182: 1426-1434.

DOI: $10.1183 / 09031936.00039411$

\section{From the authors:}

We thank S. Krüger and co-workers for their thoughtful comments and suggestions.

With regard to the Pneumonia Severity Index (PSI), most subsequent risk scores and prognostic markers in communityacquired pneumonia (CAP) research focused on 30-day mortality despite the well-known limitations of this end-point. Mortality may not be a directly related to infection, but rather due to comorbidities, advanced age or secondary complications within the follow-up period. In fact, within the Procalcitonin-Guided Antibiotic Therapy and Hospitalisation in Patients with Lower Respiratory Tract Infections (ProHOSP) study, deaths were evenly distributed within the 30-day follow-up, as demonstrated in the Kaplan-Meier plot. When restricting our analysis to short-term mortality within the first 3 days after admission, procalcitonin (PCT) showed a superior prognostic performance (area under the curve (AUC) 0.68). Also, considering not only initial but also follow-up PCT levels within our study significantly improved the prognostic performance of PCT. This was also true in a previous Legionella sp. CAP study [1] and intensive care unit (ICU) studies $[2,3]$. Therefore, we advocate the use of PCT for prognostication in an in-patient CAP setting primarily in combination with a clinical risk score that also incorporates static risk factors, such as age and comorbidities, and consideration of the kinetic of the marker over time, as nondecreasing levels point to adverse outcome.
There are several possible explanations of the difference in discrimination of PCT in our study (AUC 0.60) [4] and the previous CAPNETZ study (AUC 0.80) [5]. First, antibiotic pretreatment may affect the prognostic performance of PCT, as patients who are admitted to the hospital with secondary treatment failure show some decrease in PCT levels from its peak value, but these patients are still at high risk for an adverse outcome. However, restricting our analysis to patients without antibiotic pre-treatment did not significantly improve the prognostic performance of PCT (AUC 0.61). Secondly, due to differences in study design compared with CAPNETZ, patients in ProHOSP had, on average, greater disease severity, reflected by higher rates of in-patient treatment (94.6 versus $66.6 \%$ ), higher median PCT values of in-patients $\left(0.51\right.$ versus $\left.0.24 \mu \mathrm{g} \cdot \mathrm{L}^{-1}\right)$ and higher mortality rates (5.4 versus $4.5 \%$ ) [5, 6]. Previously, it has been shown that an initial PCT level $<0.25 \mu \mathrm{g} \cdot \mathrm{L}^{-1}$ rules out bacteraemic CAP with a $90 \%$ negative predictive value [7] and safely discriminates patients with pneumonic respiratory infection requiring antibiotics from those with bronchitis not requiring antibiotics $[6,8]$. Thus, in a lower risk population, PCT may, in fact, help to differentiate patients with CAP (and thus expected to be at higher risk) from those with non-CAP respiratory infection (and at lower mortality risk). This is also supported when looking at the prognostic performance of PCT in the earlier ProcalcitoninGuided Treatment on Antibiotic Use and Outcome in Lower Respiratory Tract Infections (ProRESP) study. In the overall cohort of 243 patients, 87 with CAP, the prognostic performance for mortality prediction of PCT was 0.78. Yet, the AUC of PCT decreased to 0.55 when only considering CAP patients within this study. Again, when considering all patients with respiratory infections in ProHOSP, the AUC of PCT was 0.65 and decreased to 0.60 when restricted to CAP patients [4]. Finally, in the Procalcitonin Guidance of Antibiotic Therapy in CommunityAcquired Pneumonia (ProCAP) study, including only CAP patients, the AUC of PCT was 0.61 [9]. These findings suggest that in lower risk settings and/or a population with mixed respiratory infection, initial PCT level may be helpful to identify CAP patients and, thereby, provide more prognostic information concerning expected mortality. In contrast, in higher risk populations, initial PCT may have limited prognostic accuracy and repeated PCT measurements are clearly preferable.

Restricting the analysis to only high risk patients (PSI class $>4$ or CURB-65 score $>2$ (confusion, urea $>7 \mathrm{mmol} \cdot \mathrm{L}^{-1}$, respiratory frequency $\geqslant 30$ breaths $\cdot \mathrm{min}^{-1}$, systolic blood pressure $<90 \mathrm{mmHg}$ or diastolic blood pressure $\leqslant 60 \mathrm{mmHg}$ and age $\geqslant 65 \mathrm{yrs})$ ) and only one PCT cut-off of $0.25 \mu \mathrm{g} \cdot \mathrm{L}^{-1}$, as suggested by $\mathrm{S}$. Krüger and co-workers, shows a better separation of survivors and nonsurvivors, particularly in the early study period, which does not, however, reach statistical significance in the overall 30-day period (fig. 1). This is also in accordance with a CAP study from Pittsburgh, PA, USA demonstrating a significant effect of low PCT to rule out mortality in high-risk CAP patients [10].

In conclusion, there is evidence that: 1) increased PCT levels of $>0.25 \mu \mathrm{g} \cdot \mathrm{L}^{-1}$ dose-dependently identify patients at higher risk for (bacteraemia) CAP in a low-risk population with mixed respiratory infections; 2) not decreasing PCT levels during follow-up despite antibiotic therapy identifies patients not responding to therapy, possibly resulting in adverse outcomes; and 3) low PCT levels of $<0.25 \mu \mathrm{g} \cdot \mathrm{L}^{-1}$ help to rule out mortality 